\title{
The Impact of Senior Mobility Funding on Hearing Aid Acquisition and Compliance to Hearing Aid Use in a Singapore Hospital
}

\author{
Kenneth Chua Wei De ${ }^{1,2}$ \\ ${ }^{1}$ Departments of Otolaryngology, ${ }^{2}$ Audiology, Changi General Hospital, Singapore
}

Received May 12, 2020

Revised June 4, 2020

Accepted September 8, 2020

Address for correspondence

Kenneth Chua Wei De, M.Sc, FAIB

Departments of Otolaryngology,

Audiology, Changi General Hospital,

2 Simei, Street 3, S529889,

Singapore

Tel +65-6936-5259

Fax +65-6936-5259

E-mail kc.weide@gmail.com
Background and Objectives: In 2013, the Singapore government reviewed and expanded the Senior Mobility Fund (SMF) to provide subsidy for assistive devices, including hearing aids (HA). While SMF has improved accessibility to HA, its impact on HA acquisition has not been determined. The study aims to elucidate the influence of SMF on HA acquisition and the relationship between financial funding and compliance to HA use. Subjects and Methods: Retrospective review of 643 patients seen between January 2017 to January 2018 at the earnose and throat specialist outpatient clinic, who were referred for a hearing aid evaluation. Of the 643 patients, 109 patients with baseline hearing handicap $(\mathrm{HH})$ scores recorded, and no formal diagnosis of cognitive impairment were included. The patients were grouped according to SMF eligibility and clinical data were obtained. Results: The odds ratio for acquiring HA was significantly higher with SMF, regardless of $\mathrm{HH}$ scores. When looking at actual degree of hearing loss (DHL), HA uptake was significantly higher in the least severe of DHL categories. There is no relationship between SMF and compliance to HA use. However, $\mathrm{HH}$ score is positively correlated with data log in regression analyses. Conclusions: SMF appears to influence $\mathrm{HA}$ acquisition. Especially in financially funded patients with low self-perceived $\mathrm{HH}$, the benefits of HA may be underappreciated given the few hours of HA use. Further studies are warranted to investigate the impact of financial funding on outcomes of HA users to help policy planners and clinicians be prudent in the utilization of SMF.

J Audiol Otol 2021;25(1):8-13

KEY WORDS: Hearing aids · Economics - Compliance · Hearing loss · Persons with hearing impairments.

\section{Introduction}

Population statistics in 2019 have estimated that $70 \%$ of the 5.7 million people in Singapore were residents comprising of both citizens and permanent residents [1]. Among the resident adults, an approximately $3 \%$ of surveyed residents (Singapore National Health Survey) have disabling hearing loss (HL) [2], which is defined by the World Health Organization as having an $\mathrm{HL}$ more than $40 \mathrm{~dB}$ in the better hearing ear. This is further estimated by a recent community study on HL amongst the elderly $(n=338)$ over 60 years old, with a dis-

This is an Open Access article distributed under the terms of the Creative Commons Attribution Non-Commercial License (https://creativecommons.org/licenses/by-nc/4.0/) which permits unrestricted non-commercial use, distribution, and reproduction in any medium, provided the original work is properly cited. abling HL prevalence of $16.2 \%$ [3]. This prevalence is specifically about $9-10 \%[3,4]$ in the age group $60-69 ; 22 \%$ for $70-79$ years old; and $36 \%$ for residents 80 years and above [3]. There are over half a million Singapore residents aged 65 and above [1] and hence an estimated over 100,000 elderly with disabling HL. The number of disabling HL is expected to increase with Singapore's ageing population, as the number of seniors age 65 and above is estimated to be over 900,000 by 2030 [5].

However, the hearing aid (HA) uptake rate remains to be extremely low from $3.3 \%$ among Singapore adults to $7.5 \%$ of the elderly, in contrast to a higher rate of HA adoption in other countries such as Japan (14\%), Germany (37\%), and the United Kingdom (48\%) [6-8]. This low HA uptake among Singaporeans is of a concern and hence, there is a need to un- 
derstand the factors involved in motivation, barriers, and compliance to hearing healthcare. A systematic review of 1,154 articles in 2016 on factors involved in access and utilization of adult hearing healthcare, identified cost of hearing healthcare: in particular, the cost of HA as a prominent factor amongst other audiological and non-audiological factors [9]. In line with this, the Singapore government reviewed and expanded the Senior Mobility Fund (SMF) to provide a 90\% subsidy for HA to eligible seniors in 2013. This has significantly reduced the cost to acquire HA and improved accessibility for the elderly with financial needs. However, to our knowledge, the impact of HA subsidy has yet to be assessed, and it is not known if financial funding has affected HA adoption rates in Singapore, as compared to other countries like the United Kingdom, where financial funding are also available and higher HA adoption rates are seen.

Hence, this study explored the influence of SMF on HA acquisition, to determine if funding resulted in higher HA uptake rate and if, there was a relationship between financial funding and compliance to HA use.

\section{Subjects and Methods}

\section{Participants}

A retrospective analysis of 643 HA patients seen at the outpatient Otorhinolaryngology Ear-Nose-Throat (ENT) clinic at Changi General Hospital from January 2017 to January 2018 was performed. We reviewed the clinical data of patients seen for hearing aid evaluation (HAE) from our proprietary hearing aid software NOAH to extract demographic and clinical data. The information was exported to an excel sheet and stripped of all personal identifiers to anonymize the participants. Of the 643 patients seen in this period, 113 patients who had their baseline hearing handicap $(\mathrm{HH})$ scores recorded during HAE or pre-hearing aid fitting (pre-HAF) were selected. Four patients who were formally diagnosed by either a trained psychiatrist or geriatrician with Alzheimer or dementia were excluded, as their judgment on HA acquisition was compromised. One hundred nine patients were eventually included and were assigned to two groups, depending on SMF eligibility. The groups were further analyzed, with stratification according the degree of $\mathrm{HH}$ as recorded on the Hearing Handicap Inventory Adult/Elderly-Short (HHIA/E-S), as well as the actual degree of hearing loss (DHL).

\section{Ethics statement}

Written informed consent was obtained from included participants and all study protocols adhered to the guidelines and regulations which, met the standards of the Helsinki Declara- tion of 1975. This study was exempted from local Singhealth Centralised Institutional Review Board (CIRB) approval due to anonymity of the data and participants.

\section{Definition of SMF eligibility}

Singapore citizens aged 60 and older with a household monthly income per person of up to $\$ 2,000$ or an annual value of residence of up to $\$ 13,000$ for households without income. Patients who are eligible for SMF will be entitled up to $\$ 2,700$ for a pair of prescribed HA. Patients must also have an equal to or more than $40 \mathrm{~dB}$ in the pure-tone averages (PTA) of $0.5,1,2$, and $4 \mathrm{kHz}$ in at least one ear to qualify for SMF. This PTA criteria also includes patients with a unilateral hearing loss, with a $25 \mathrm{~dB}$ or better PTA in the better ear.

\section{Description and categorization of HHIA/E-Short scores}

The HH Inventory is a self-assessment scale, composed of two subscales (social and emotional), used to assess for functional impairments of HL. A 25-item questionnaire is administered to adults who are still in employment, while a shortened 10 -item questionnaire is used for the elderly without employment. Both questionnaires have high Cronbach's alpha scores with good test re-test reliability [10,11]. The HHIA and HHIE$\mathrm{S}$ is scored upon 100 and 40, respectively. A cut-off score of 30 and 17 were determined for up to a mild degree of $\mathrm{HH}$. This cut-off is the median score based on the range of $18-42$ (HHIA; mild to moderate) and 10-24 (HHIE-S; mild to moderate) as determined by the original authors of the handicap questionnaires. Patients with up to a mild degree of $\mathrm{HH}$ were categorized as negative handicap (-), while those with a moderate degree of $\mathrm{HH}$ and beyond were assigned as positive $(+)$.

\section{Categorization of $\mathrm{DHL}$}

Audiograms were classified into 1) sloping mild to moderately severe/profound, 2) moderate to profound and 3) severe to profound sensorineural HL with decreasing gradient of slope as DHL increased.

\section{Statistical analysis}

The data was analyzed using Statistical Package for Social Sciences (SPSS 21.0; IBM Corp., Armonk, NY, USA). We first performed an independent sample t-test and a 2 by 2 crosstabulation to assess if there are significant differences in the mean age between gender and distribution of gender between the SMF eligible and non-SMF eligible groups. Chi-square analyses were further performed to establish the relationship between categorical variables, namely SMF eligibility and HA acquisition. The chi-square analyses were further stratified accordingly to category of $\mathrm{HH}$ and actual DHL to further eluci- 
date the relationship between SMF eligibility and decision to acquire HA. Logistic regression analysis was performed to analyze if age, gender, SMF eligibility, category of $\mathrm{HH}$ and category of DHL are predictors of HA compliance, denoted by hours of hearing aid use on the recorded data log. When the actual scaled numerical values of $\mathrm{HH}$ and DHL were considered, the relationship between HA compliance and its independent variables were further elucidated with linear regression analysis. All assumptions of regression analysis such as multicollinearity, homoscedasticity, normality, and independence of residuals were fulfilled and checked prior to regression analyses.

\section{Results}

There were 65 males and 44 females with an overall median age of 76 years. Of the 109 patients, 61 patients were SMF eligible and 48 were not; 72 (66.1\%) eventually acquired HA, while 37 (33.9\%) did not. Demographic and clinical details can be found in Table 1. There was no significant difference in the median age $(p=0.60)$ or distribution of gender between groups ( $p=0.45$ ). Of the patients, $80.3 \%$ (49/61) with SMF acquired HA and were $4.44 \times$ more likely to acquire HA as compared to their non-SMF funded peers (HA uptake rate: 47.9\%; 23/48), with a significant relationship between SMF financial funding and decision to acquire HA. This significance also had a moderate effect size (Table 2).

When the patients were stratified according to the severity of the handicap, SMF funded patients with a moderate to severe $\mathrm{HH}$ were $18 \times$ more likely to acquire $\mathrm{HA}(96.8 \% ; 30 / 31)$, as compared to non-SMF funded patients $(62.5 \% ; 10 / 16)$; similarly with a statistically significant relationship $(p<0.001$, Fisher-exact two sided) and large effect size between SMF funding and HA acquisition (Table 2). Conversely, when $\mathrm{HH}$ is absent or mild, the SMF funded patients are still $2.52 \times$ more likely to purchase HA $(63.3 \% ; 19 / 30)$, compared to patients without SMF funding $(40.6 \% ; 13 / 32)$ despite less sig- nificance $(p=0.07)$ (Table 2$)$. SMF hence, appears to be pivotal in HA acquisition regardless of $\mathrm{HH}$ severity.

As the HH questionnaire is more specific than sensitive [3], it may not correlate well with the DHL. Some patients may have a severe HL with a low self-reported $\mathrm{HH}$ and vice-versa. Hence, we looked at the actual DHL and categorized the patients according to their HL severity as defined previously $(1-3)$. We see that patients are $12.5 \times$ more likely to acquire HA $(71.4 \%$; 10/14) when they are eligible for SMF in the least severe of DHL category (1), with a large effect size and a statistically significant relationship $(p<0.001)$ between HL severity and HA acquisition (Table 3). This is in comparison

Table 2. The association between SMF eligibility and HA acquisition according to degree of $\mathrm{HH}$

\begin{tabular}{|c|c|c|c|c|}
\hline & $\begin{array}{c}\mathrm{HA} \\
\text { acquisition } \\
\text { (no) }\end{array}$ & $\begin{array}{c}\text { HA } \\
\text { acquisition } \\
\text { (yes) }\end{array}$ & Total & $p$-value \\
\hline Overall patients* & & & & $<0.001$ \\
\hline SMF (no) & 25 & 23 & 48 & \\
\hline SMF (yes) & 12 & 49 & 61 & \\
\hline Total & 37 & 72 & 109 & \\
\hline $\mathrm{HH}(+)^{\dagger}$ & & & & $<0.001$ \\
\hline SMF (no) & 6 & 10 & 16 & \\
\hline SMF (yes) & 1 & 30 & 31 & \\
\hline Total & 7 & 40 & 47 & \\
\hline $\mathrm{HH}(-)^{\phi}$ & & & & 0.070 \\
\hline SMF (no) & 19 & 13 & 32 & \\
\hline SMF (yes) & 11 & 19 & 30 & \\
\hline Total & 30 & 32 & 62 & \\
\hline
\end{tabular}

$2 \times 2$ chi-square cross tabulation. *seniors with SMF are $4.44 \times$ more likely to acquire hearing aids as compared to seniors without SMF. ${ }^{\dagger}$ moderate to severe $\mathrm{HH}(\mathrm{p}<0.001$, with a moderate effect size); seniors with a moderate to severe $\mathrm{HH}$ are $18 \times$ more likely to acquire hearing aids as compared to seniors with mild or better $\mathrm{HH}$ scores $(p<0.001$, with a large effect size). " no HH to mild handicap; seniors with mild or better $\mathrm{HH}$ scores are $2.52 \times$ more likely than seniors with moderate and beyond $\mathrm{HH}$ severity to acquire hearing aids ( $p=0.07$, with a small effect size). SMF: Senior Mobility Fund, HH: hearing handicap, HA: hearing aid

Table 1. Demographic and clinical information of included participants

\begin{tabular}{lccc}
\hline \multicolumn{1}{c}{ Variable } & SMF, eligible $(\mathrm{n}=61)$ & SMF, not eligible $(\mathrm{n}=48)$ & Overall $(\mathrm{n}=109)$ \\
\hline Age (yr), median (range) & $76.0(23-93)$ & $76.5(42-97)$ & $76.0(23-97)$ \\
Male:female & $41: 20$ & $24: 24$ & $65: 44$ \\
Category of hearing handicap (+ve:-ve)* & $31: 30$ & $16: 32$ & $47: 62$ \\
Hearing aid acquisition (yes:no) & $49: 12$ & $23: 25$ & $72: 37$ \\
Degree of hearing loss $(1: 2: 3)^{\dagger}$ & $14: 35: 12$ & $18: 26: 4$ & $32: 61: 16$ \\
Data log (hr), median & 1.1 & 1.0 & 1.0 \\
\hline
\end{tabular}

*positive category (+ve) refers to patients with a moderate and beyond hearing handicap score; negative category (-ve) refers to patients with a mild degree and better hearing handicap score. ${ }^{\dagger}$ degree of hearing loss $(1: 2: 3)$ : category 1 , mild to moderately severe/profound hearing loss; category 2, moderate to severe hearing loss; category 3 , severe to profound hearing loss. SMF: Senior Mobility Fund 
Table 3. The association table between SMF eligibility and HA acquisition, stratified by categories of $\mathrm{DHL}$

\begin{tabular}{ccc}
\hline HA & HA & Sub-total \\
acquisition (no) & acquisition (yes) & \\
\hline
\end{tabular}

DHL (1): sloping mild to moderately severe/profound hearing loss

$\begin{array}{lrrr}\text { SMF (no) } & 15 & 3 & 18 \\ \text { SMF (yes) } & 4 & 10 & 14 \\ \text { Sub-total } & 19 & 13 & 32\end{array}$

*Chi-square: $9.79, p<0.001$, odds ratio $=12.5$

DHL (2): moderate to profound hearing loss

$\begin{array}{lrcc}\text { SMF (no) } & 8 & 18 & 26 \\ \text { SMF (yes) } & 5 & 30 & 35 \\ \text { Sub-total } & 13 & 48 & 61 \\ & & { }^{+} \text {Chi-square: } 2.42 & p=0.1\end{array}$

DHL (3): severe to profound hearing loss

$\begin{array}{cccc}\text { SMF (no) } & 2 & 2 & 4 \\ \text { SMF (yes) } & 3 & 9 & 12 \\ \text { Sub-total } & 5 & 11 & 16 \\ & & { }^{\dagger} \text { Chi-square: } 0.87, p=0.35 \\ \text { Total } & 37 & 72 & 109\end{array}$

$2 \times 2$ chi-square cross tabulation. *significant $p<0.001$, with a large effect size. Seniors in the least severe of hearing loss category are $12.5 \times$ more likely to acquire hearing aids when they are eligible for SMF as compared to seniors without SMF. ${ }^{\dagger}$ less significant $p=0.12$, with small effect size for seniors with moderate to profound hearing loss; SMF eligibility did not significantly influence hearing aid acquisition. " not statistically significant, $p=$ 0.35. SMF eligibility did not influence seniors with the most severe degree of hearing loss in decision to acquire hearing aids. $\mathrm{HH}$ : hearing handicap, SMF: Senior Mobility Fund, HA: hearing aid, DHL: degree of hearing loss

with non-SMF funded patients with only $16.7 \%$ (3/18) patients who acquired HA. However, the influence of SMF is not significant in the other two DHL categories (2 and 3).

Of the 72 patients who acquired HA, we analyzed the hours of hearing aid use (data $\log$ ) to assess if SMF eligibility, $\mathrm{HH}$, DHL, age and gender predicted data log. Data log hours were categorized into less than 4 hours $(0)$ and more than 4 hours of use (1). Age was recoded to less than (0) or more than (1) 65 years old. The logistic regression showed that only $\mathrm{HH}$ was a significant predictor of data log hours with statistical significance $(p<0.001)$. Holding all other independent variables constant, patients with up to a mild degree of $\mathrm{HH}$ are $39.36 \times(95 \%$ confidence interval; 8.86-174.93) more likely to use their hearing aids for less than 4 hours (Table 4). This is further supported by linear regression analysis when we looked at the scaled numerical value of data log hours (dependent variable) versus actual $\mathrm{HH}$ scores (independent variable).

For every one-point increase in HH on the HHIE-S, there is a corresponding 0.18 hours (11 minutes) increase in hearing aid use, holding all other independent variables constant (Fig. 1). This is statistically significant $(p<0.001)$ with a $95 \%$ confidence interval $(0.11-0.25)$ and a moderate positive rela-
Table 4. Logistic regression analysis

\begin{tabular}{|c|c|c|}
\hline Data log (hours) & $p$-value & Odds ratio (exp B) \\
\hline$[S M F 0 / 1]^{*}$ & 0.34 & 2.22 \\
\hline 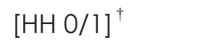 & $<0.001$ & 39.36 \\
\hline$[\mathrm{HL} \text { Cat } 1 / 2 / 3]^{\dagger}$ & 0.55 & 2.10 \\
\hline$[\text { Gender } \mathrm{F} / \mathrm{M}]^{\S}$ & 0.48 & 0.59 \\
\hline$[\text { Age } 0 / 1]^{\|}$ & 0.61 & 1.61 \\
\hline \multicolumn{3}{|c|}{$R^{2}=0.44$, adjusted $R^{2}=0.59$} \\
\hline
\end{tabular}

*SMF: Senior Mobility Fund (0: not eligible, 1 : eligible). ${ }^{\dagger} \mathrm{HH}$ : hearing handicap (0, mild or better hearing handicap scores; 1 , moderate and beyond hearing handicap scores). "HL Cat: hearing loss category (1, mild to moderate severe hearing loss; 2 , moderate to severe hearing loss; 3 , severe to profound hearing loss). ${ }^{\S}$ Gender: $F$, female; $M$, male. "Age (0: less than 65 years of age, 1: more than 65 years of age). Holding all other variables constant, seniors with mild of better degree of hearing handicap are $39.36 \times$ more likely to use their hearing aids for less than 4 hours on average in a week. The predictors explain $59 \%$ of the variance in data.

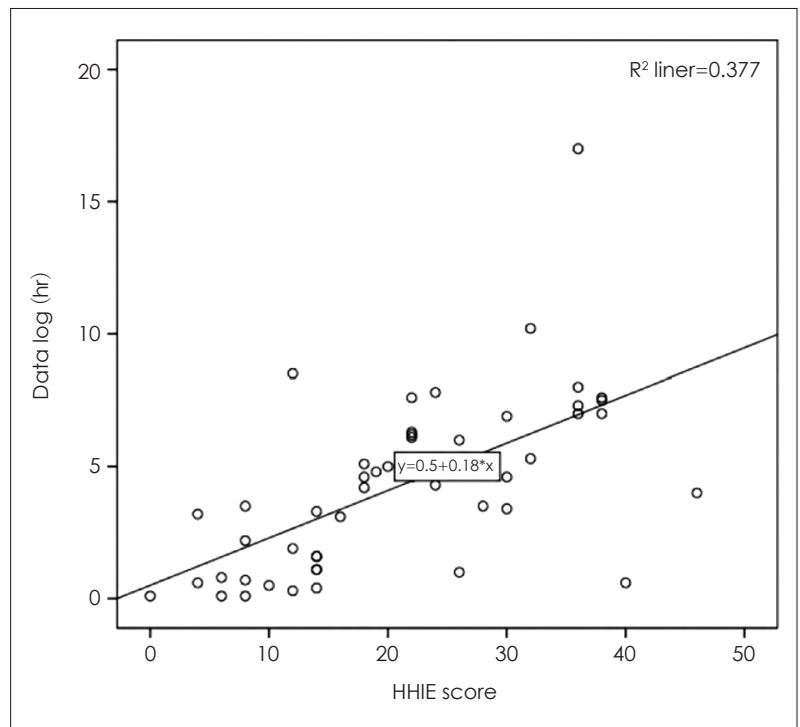

Fig. 1. Scatterplot of data log (hours of hearing aid use) vs. HHIE socres. For every one-point increase in hadicap score, there is a corresponding 0.18 hours (11 minutes) increase in hearing aid use, with a significant $(p<0.001)$ correlation of $0.6(60 \%)$ and data explaining $37.7 \%$ of the variance. HHIE: Hearing Handicap Inventory Elderly.

tionship (Pearson correlation of 0.6). Similarly, for every onepoint increase in $\mathrm{HH}$ on the HHIA, there is a statistically significant ( $p=0.01$ ) increase in 0.12 hours ( 7 minutes) of hearing aid use (95\% confidence interval; $0.03-0.21)$ and a similar moderately strong positive relationship (Fig. 2).

\section{Discussion}

There is significant influence of SMF on HA acquisition. A pair of hearing aids at the basic platform level cost between $\$ 2,500-\$ 3,200$ Singapore dollars, which is an exorbitant 


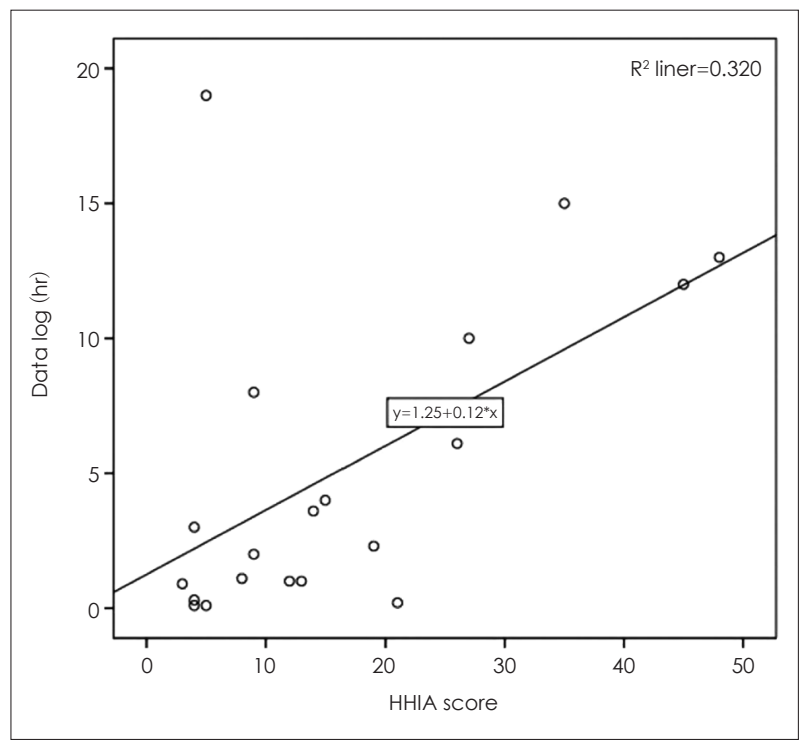

Fig. 2. Scatterplot of data log (hours of hearing aid use) vs. HHIA scores. For every one-point increase in hadicap scores, there is an increase in 0.12 hours ( 7 minutes) of hearing aid use. This is a significant $(p=0.01)$ correlation of $0.6(60 \%)$ with data explaining $32 \%$ of the variance. HHIA: Hearing Handicap Inventory Adult.

amount for most patients. Amongst other factors, affordability due to socio-economic status particularly cost of hearing aids was identified in the literature to be a major barrier in HA acquisition [12-15]. Hence, it is not surprising that SMF eligibility predicted HA acquisition. This is evident regardless of $\mathrm{HH}$ severity, which suggested that when $\mathrm{HH}$ scores were low, patients with SMF have a greater propensity towards acquiring HA. Conversely, when self-perceived HH was significant, SMF eligibility became a barrier to HA acquisition.

However, because of self-recognition and acceptance of HL, self-reported HH may not correlate with DHL. When we looked at the DHL, SMF eligibility was not a significant factor in HA acquisition for DHL of a moderate degree and beyond (2 and 3 ). However, in the least severe DHL category (1), SMF eligible patients are $12.5 \times$ more likely to acquire HA.

While SMF has improved affordability and hence, accessibility to HA, the cost must be justified. The current SMF eligibility criteria does not take into account patients' functional impairments and may hence not be stringent enough. Of the 30 patients in the least DHL category, more than 70\% (23/32) had no or mild self-reported HH scores. A further 35\% (8/23) of these patients acquired hearing aids, and more than $50 \%$ of them $(5 / 8)$ had SMF.

$\mathrm{HH}$ score perceived at baseline has been studied to be a significant factor in compliance to hearing healthcare recommendations [16]. In this study, although there was no significant relationship between SMF eligibility and compliance to HA use, self-reported $\mathrm{HH}$ was a significant predictor of data log hours. Regression analysis suggested that self-reported $\mathrm{HH}$ was positively correlated with the hours of hearing aid use. Since most of the patients with the least severe DHL had low HH scores (median=9), their sub-optimal hours of HA (median $=0.9$ hours) use may not justify the utilization of SMF. Hence, in this group of patients, there may be an overconsumption of public funding.

This study was a single-site retrospective investigation, whose study design cannot be controlled. The data hence cannot be extrapolated for the general population. Further prospective multi-site studies are warranted. This study only investigated the influence of funding and hence cost on hearing aid acquisition. However, a systematic review [9] of the literature has suggested that cost is not the only factor. There are other factors not considered here such as; self-perception of benefit of hearing [17], family/social-support [18], personality [19], self-efficacy [20], anxiety disorders [21], stigma [22], social identity [23], family and friends' shared experiences [24], coexisting health conditions [25] and cultural/racial/ethnic factors [24] amongst others.

Where data-log of the HA were concerned, it may be an overestimation if patients had forgotten to remove their HA batteries or an underestimation if batteries were not changed periodically. There are also other factors of HA compliance aside from perceived $\mathrm{HH}$, not considered such as, education level [16], socio-economic status [26], positive support from significant other [27] and self-efficacy [25].

In conclusion, SMF seems to have significantly influenced HA acquisition, as cost may be a greater concern than selfreport $\mathrm{HH}$ in determining hearing aid acquisition. However, the benefits of hearing aids may be underappreciated for SMF funded patients with low self-perceived $\mathrm{HH}$, who acquired HA. This is evidently seen in the significantly fewer hours of hearing aid use in this group of patients. Further studies are warranted to compare the clinical and functional HA outcomes between patients with and without SMF funding. The utilization of SMF may not be well justified in SMF funded patients with low self-perceived $\mathrm{HH}$ and hence, may be an overconsumption of public funds.

\section{Acknowledgments}

The author would like to thank the Departments of Otolaryngology and Audiology for their support in this study.

\section{Conflicts of interest}

The author has no financial conflicts of interest.

\section{ORCID iD}

Kenneth Chua Wei De https://orcid.org/0000-0002-2677-1183 


\section{REFERENCES}

1) Ministry of Trade and Industry, Department of Statistics. Population trends, 2019 [serial online]. Singapore: Ministry of Trade \& Industry; 2019 September [cited 2020 May 11]. Available from: https:// www.singstat.gov.sg/-/media/files/publications/population/population2019.pdf.

2) Ministry of Health Singapore, Epidemiology and Disease Control Division. National health survey 2010 [Internet]. Singapore: Ministry of Health Singapore; 2011 October [cited 2020 May 11]. Available from: https://www.moh.gov.sg/docs/librariesprovider5/resources-statistics/reports/nhs2010---low-res.pdf.

3) Lee JC, Danker AN, Wong YH, Lim MY. Hearing loss amongst the elderly in a Southeast Asian population-a community-based study. Ann Acad Med Singap 2017;46:145-54.

4) Ho EC, Zhang H, Ong WMW, Li K, Bei YTE, Medapati SVR, et al. Hearing impairment and hearing aid usage in Singapore. Int J Audiol 2018;57:291-301.

5) Prime Minister's Office, National Population and Talent Division. Population White Paper: a sustainable population for a dynamic Singapore [Internet]. Singapore: Prime Minister's Office; 2013 January [cited 2020 May 11]. Available from: https://www.strategygroup.gov.sg/media-centre/population-white-paper-a-sustainablepopulation-for-a-dynamic-singapore.

6) Chien W, Lin FR. Prevalence of hearing aid use among older adults in the United States. Arch Intern Med 2012;172:292-3.

7) European Hearing Instrument Manufacturers Association. EuroTrak UK 2009 [Internet]. Zürich: Anovum GmbH; C2009 [cited 2020 May 11]. Available from: https:/www.ehima.com/wp-content/ uploads/2014/03/protected_eurotrak_2009_uk_pr.pdf.

8) European Hearing Instrument Manufacturers Association. JapanTrak 2012 [Internet]. Zürich: Anovum GmbH; C2012 [cited 2020 May 11]. Available from: http://www.ehima.com/wp-content/uploads/2014/03/protected_japantrak_2012.pdf.

9) Barnett M, Hixon B, Okwiri N, Irungu C, Ayugi J, Thompson R, et al. Factors involved in access and utilization of adult hearing healthcare: a systematic review. Laryngoscope 2017;127:1187-94.

10) Newman CW, Weinstein BE, Jacobson GP, Hug GA. The hearing handicap inventory for adults: psychometric adequacy and audiometric correlates. Ear Hear 1990;11:430-3.

11) Ventry IM, Weinstein BE. Identification of elderly people with hearing problems. ASHA 1983;25:37-42.

12) Popelka MM, Cruickshanks KJ, Wiley TL, Tweed TS, Klein BE, Klein R. Low prevalence of hearing aid use among older adults with hearing loss: the epidemiology of hearing loss study. J Am Geriatr
Soc 1998;46:1075-8.

13) Kochkin S. MarkeTrak VII: obstacles to adult non-user adoption of hearing aids. Hearing J 2007;60:24-51.

14) Fischer ME, Cruickshanks KJ, Wiley TL, Klein BE, Klein R, Tweed TS. Determinants of hearing aid acquisition in older adults. Am J Public Health 2011;101:1449-55.

15) Houggard S, Ruf S, Egger C. EuroTrak + JapanTrak 2012: societal and personal benefits of hearing rehabilitation with hearing aids. Hearing Rev 2013;20:16-26.

16) Mulrow CD, Tuley MR, Aguilar C. Correlates of successful hearing aid use in older adults. Ear Hear 1992;13:108-13.

17) van den Brink RH, Wit HP, Kempen GI, van Heuvelen MJ. Attitude and help-seeking for hearing impairment. Br J Audiol 1996;30:31324.

18) Duijvestijn JA, Anteunis LJ, Hoek CJ, Van Den Brink RH, Chenault MN, Manni JJ. Help-seeking behaviour of hearing-impaired persons aged $\geq 55$ years; effect of complaints, significant others and hearing aid image. Acta Otolaryngol 2003;123:846-50.

19) Cox RM, Alexander GC, Gray GA. Who wants a hearing aid? Personality profiles of hearing aid seekers. Ear Hear 2005;26:12-26.

20) Meyer C, Hickson L, Fletcher A. Identifying the barriers and facilitators to optimal hearing aid self-efficacy. Int J Audiol 2014;53 Suppl 1:S28-37.

21) Meyer C, Hickson L, Lovelock K, Lampert M, Khan A. An investigation of factors that influence help-seeking for hearing impairment in older adults. Int J Audiol 2014;53:S3-17.

22) Wallhagen MI. The stigma of hearing loss. Gerontologist 2010;50: 66-75.

23) Claesen E, Pryce H. An exploration of the perspectives of help-seekers prescribed hearing aids. Prim Health Care Res Dev 2012;13:27984.

24) Laplante-Lévesque A, Hickson L, Worrall L. Factors influencing rehabilitation decisions of adults with acquired hearing impairment. Int J Audiol 2010;49:497-507.

25) Öberg M, Marcusson J, Nägga K, Wressle E. Hearing difficulties, uptake, and outcomes of hearing aids in people 85 years of age. Int $\mathrm{J}$ Audiol 2012;51:108-15.

26) Francis HW, Yeagle JA, Thompson CB. Clinical and psychosocial risk factors of hearing outcome in older adults with cochlear implants. Laryngoscope 2015;125:695-702.

27) Hickson L, Meyer C, Lovelock K, Lampert M, Khan A. Factors associated with success with hearing aids in older adults. Int J Audiol 2014;53 Suppl 1:S18-27. 